\title{
Diet composition of Indian flying fox (Pteropus giganteus) in Kathmandu Valley
}

\author{
Nabina Tiwari ${ }^{1{ }^{\star}}$, Narayan Prasad Koju², Pushpa Raj Acharya ${ }^{3}$, Man Kumar Dhamala ${ }^{1}$ \\ ${ }^{1}$ Central Department of Environmental Science, Tribhuvan University, Kathmandu, Nepal. \\ ${ }^{2}$ Center for Postgraduate Studies, Nepal Engineering College, Pokhara University, Kathmandu, Nepal. \\ ${ }^{3}$ Central Campus of Science and Technology, Faculty of Science \& Technology, Mid-Western University, Surkhet, Nepal.
}

\begin{abstract}
The composition of diet reflects the food availability and food preference by wildlife in their respective habitat. Flying fox (Pteropus giganteus) is the largest bat species that inhabit the urban area of Kathmandu Valley. The diet composition of Flying fox and their conservation threat was studied from January to April 2018. Bats roosting at Kesharmahal, Kathmandu and Sallaghari, Bhaktapur were selected for the study. The diet consumed was explored by micro-histological analysis from fresh fecal samples (guano) and bolus collected from the roost site. The samples were collected by spreading plastic sheets of $2 \mathrm{~m} \times 2 \mathrm{~m}$ for a whole day. The droppings from bats were collected on $50 \mathrm{~mL}$ vials with $70 \%$ alcohol. Altogether 140 samples were collected. The collected droppings were used to micro histological slides where the seeds were isolated and identified using a hand lens. The pollens presence in slides were observed under the compound microscope and tallied with the reference slides for identification. Altogether 17 and 10 different plant families were identified from dropping of bats from Kesharmahal and Sallaghari, respectively. Among them, Moraceae and Myrtaceae were common diets in bats of both locations. Our result shows $P$. giganteus consumes food from the Myrtaceae, Brassicaceae, Malvaceae, Apiaceae, Pinaceae, Fagaceae, Proteaceae and Anacardiaceae families in Kathmandu Valley. P. giganteus mostly depend on foods present nearby the roost and also flies far away in search of food, which is comparatively minimal.
\end{abstract}

Keywords: Diet Composition, Fecal analysis, Pollination, Seed dispersal

\section{Introduction}

Bats are the unique only flying mammal, belonging to the order 'Chiroptera'. They are widely distributed and recorded throughout the world, excepting Arctic and Antarctica and some isolated oceanic islands (Mickleburgh et al., 1992). Globally about 1,117 species of bats are reported, and 128 species are recorded from south Asia (Srinivasulu et al., 2010). Fifty species of bat are reported from Nepal (Thapa, 2014), which is equivalent to $4.48 \%$ of the world bat diversity and over $40.6 \%$ of South Asia's bat diversity (Acharya et al., 2012). Nepal harbors five species of fruit bats, namely: Indian flying fox (Pteropus giganteus), short-nosed fruit bat (Cynopterus sphinx), Leschenault's Rousette (Rousettus leschenaultii), the dawn bat (Eonycteris spelaea) and Blanford's fruit bat (Sphaerias blanfordii) (Acharya et al., 2012). It is distributed in Bangladesh, Bhutan, China, India, Maldives, Myanmar, Nepal, Pakistan and Sri Lanka (Jnawali et al., 2011). Pteropus giganteus is the largest fruit bat in Nepal (Koju \& Chalise, 2010). It is widely distributed across most of Nepal, Kathmandu Valley, Jhapa, Saptari, Itahari Sunsari and Biratnagar in the eastern lowlands, Kaski, Dang, Kapilvastu and Palpa District (Acharya et al., 2012; Jnawali et al., 2011; Koju \& Chalise, 2010; Manandhar et al., 2017). They are pollinators and seed dispersers, which play a major role in forest regeneration across longer distances (Fujita \& Tuttle, 1991; Kasso \& Balakrishnan, 2013; Muscarella \& Fleming, 2007). Despite having substantial ecological benefits of bats, negative attitudes persist among people. Flying foxes are regarded as an agricultural pest and even killed (Aziz et al., 2016; Lewis \& Harrison, 1962). Based on people perception, bats in Nepal are threating from habitat destruction, food scarcity, pollution and misconception like bats meat can treat diseases like cancer (Jha, 2014; Manandhar et al., 2017).

Fruit bats feed exclusively on plant material, including fruit pulp, leaves, pollen, and nectar. Fruits are consumed with large grinding teeth, and the juice and pulp are extracted by crushing the fruit against the palate (Voigt et al., 2009). The diet of Pteropus varies with the seasonal availability of fruits (Stier \& Mildenstein, 2005). But we have very little knowledge of the diet of flying fox living inside the core urban area.

The flying fox is categorized as a mammal of the least concern by IUCN. Since less importance was given regarding conservation and there are only a few researches in this species, and in particular, diet researches are very scarce in Nepal. Therefore, the study aims to explore the diet composition in Kathmandu Valley.

A large proportion of the agricultural land, fruit trees and forest in the Kathmandu valley have been degraded due to haphazard urbanization (Ishtiaque et al., 2017). This condition might have created complexity for fruit bats in

•Corresponding author: tiwarina123@gmail.com 
searching for food nearby. The matter of concern is, do they feed on few fruits available nearby or consume a variety of fruits in garden or farm or travel far away in search of food? There is a need to explore food consumed by them in urban areas to know their feeding status. This study was, therefore, aimed at finding out the food preference of $P$. giganteus. Hence, this study would fulfill the information gap and provide a reference for further research on this.

\section{Materials and Methods} Study area

The study was conducted in two different bat roosting sites of Kathmandu valley. Kathmandu valley lies in BagmatiProvince, covering $570 \mathrm{~km}^{2}$ with an elevation ranging between $1300 \mathrm{~m}$ and $2760 \mathrm{~m}$ above sea level. Roosting site Sallaghari (27.6735 N and 85.41181 E) lies in Bhaktapur within the Army School hill forest premises with a famous

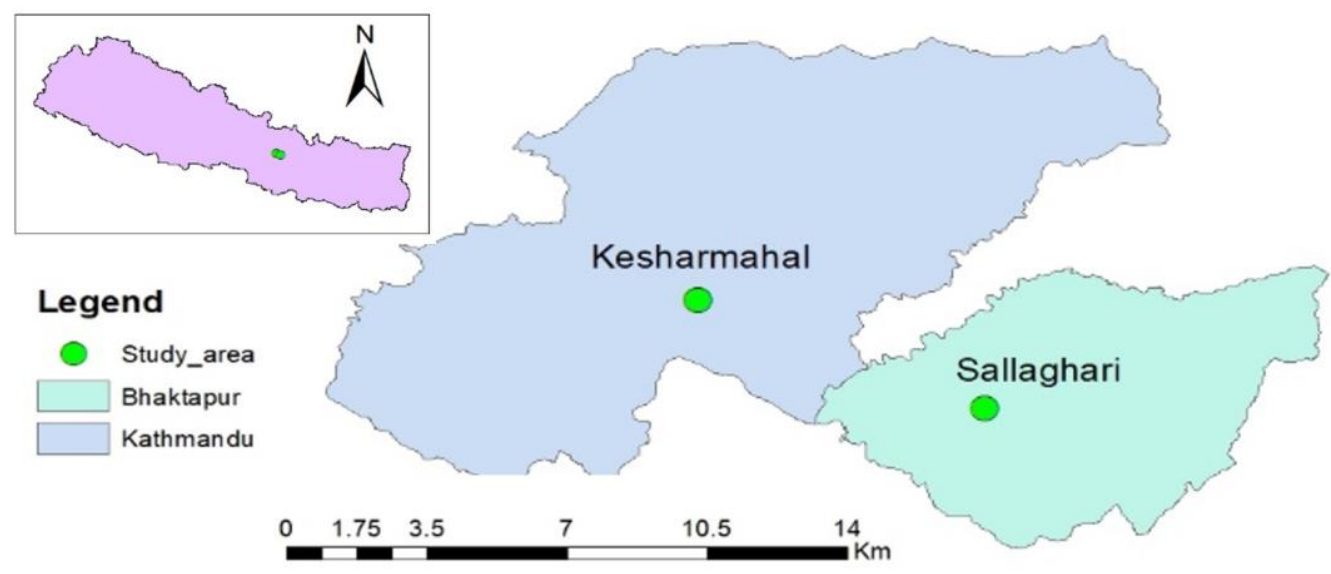

Figure 1 Map of the study area, Kesharmahal and Sallaghari, within the Kathmandu Valley

Ganesh Temple where people visit daily for recreation (picnic). Kesharmahal (27.71533 N and 85.31517 E) lies inside the previous Royal Palace nearby one of the busiest roads of Kathmandu, surrounded by tall concrete buildings at the core of Kathmandu. Both study sites lie in the core urban areas of the valley (Fig. 1).

\section{Sampling and analysis}

The study was conducted from January to April 2018. Fresh bolus and guano under the roosting site were collected by using a plastic sheet. Four plastic sheets of size $2 \mathrm{~m} \times 2 \mathrm{~m}$ were laid once a month for a whole day in each roosting site. The dropping (fecal) collected in plastic sheet was collected for further analysis. Fecal samples were collected in airtight $50 \mathrm{~mL}$ vials and preserved using 70\% alcohol. In total, 120 fecal samples (60 each site) and 20 boluses (10 each site) were collected for analysis. Reference samples of potential plant species were also collected for identification. Possible flowers and fruit samples were collected as reference samples from Kathmandu valley. Pollens present on fecal samples were tallied with the reference slides based on morphological characteristics, i.e., shape, size, symmetry, and pollen's apertures. The conventional dietary analysis method was done to identify food items following Voigt et al. (2009). In the laboratory, the seeds, pollen, culled parts of fruits, flowers and leaves were isolated from bolus and guano. The seeds were identified by visual inspection and by using a simple hand lens. Pollens were observed under the compound microscope after mounting in glass slides.

Micro histological slides were prepared and analyzed following Aziz et al. (2017). The fecal sample was mixed with hot water in a proportion of 1:2 and dissolved before the slide preparation. The sample was then placed in a test tube and centrifuged for 10 minutes at 2000 RPM (Revolutions Per Minute). The supernatant liquid was decanted, and sediment was transferred to another tube. Once again, hot water was added and centrifuged for 5 minutes. The liquid was decanted, and the bottom solid was placed onto a microscope slide and dried in an oven. A drop of warmed fuchsin stain jelly was added on the slide using a glass rod and observed under 40X magnification in a compound microscope. The seeds and pollens were identified with the help of reference collection as well as expert consultation.

The data obtained were expressed in terms of frequency of occurrence (FO) for each plant species to explore the food preferred by $P$. giganteus.

$$
\text { Frequency of Occurence }(F O)=\frac{\text { numbers of samples in which particular plant species occured }}{\text { Total mumber of samples studied }} \times 100 \%
$$




\section{Results and Discussion}

The diet of $P$. giganteus mainly consisted of fruits and flowers. Therefore, pollens of flowers and seeds of fruits were found on the fecal sample. Fruits and flowers from 17 and 10 plant families were identified in the droppings collected from the roosting site of Kesharmahal and Sallaghari, respectively. There was a minimum of 21 species of plants from the 17 families; seven species were recorded in January, seven in February, nine in March and nine in April. None of them were available in all four months, but two fig species were found from February to April.

Similarly, at Sallaghari, a minimum of 15 species of plants were recorded from 10 families; among them, 4, 10, 7 and 8 species were recorded respectively in January, February, March and April. None of them were recorded in all four months, but two species of figs and one species from Brassicaceae were common from February to April (Table 1). Plants from the family Proteaceae, Pteridophytes c.f, Tiliaceae and Syzgium cumini from the Myrtaceae family were only recorded as food consumed by bats from Sallaghari Bhaktapur. Similarly, plants from family, Anacardiaceae, Asteraceae, Betulaceae, Chenopodeaceae, Liliaceae, Myrtaceae (Psidium guajava), Oleaceae, Poaceae and Rhamnaceae are only recorded in droppings of bats from Kesharmahal. The result shows the diversity of foraging species for bats from Kesharmahal is higher than Sallaghari every month of the study period.

Table 1 Diet composition in droppings of $P$. giganteus in different months

\begin{tabular}{|c|c|c|c|c|}
\hline Family & Plant Species & Source & $\begin{array}{l}\text { Month } \\
\text { Kesharmahal }\end{array}$ & Sallaghari \\
\hline Anacardiaceae & Mangifera indica & Flower & Mar & \\
\hline Apiaceae & & Flower & Feb & Feb \\
\hline Asteraceae & & Flower & Mar & \\
\hline Betulaceae & Corylus spp. & Flower & Apr & \\
\hline Brassicaceae & & Flower & Jan, Feb, Mar & Feb, Mar, Apr \\
\hline Chenopodeaceae & & Flower & Apr & \\
\hline Fagaceae & Quercus spp. & Flower & Jan, Feb & Jan, Mar \\
\hline Liliaceae & & Flower & Mar & \\
\hline Malvaceae & Bombax ceiba & Flower & Mar, Apr & Feb, Mar \\
\hline \multirow[t]{4}{*}{ Myrtaceae } & Psidium guajava & Flower & Jan & \\
\hline & Eucalyptus camaldulensis & Flower & Mar, Apr & Feb, Mar \\
\hline & Syzgium cumini & Flower & & Mar \\
\hline & Callistemon spp. & Flower & Apr & Apr \\
\hline Oleaceae & & Flower & Jan & \\
\hline Pinaceae & Pinus roxburghii & Flower & Jan & Jan, Feb, Apr \\
\hline Poaceae & & Flower & Feb, Mar & \\
\hline Proteaceae & Grevillea robusta & Flower & Apr & Apr \\
\hline Rhamnaceae & & Flower & Jan & \\
\hline \multirow[t]{4}{*}{ Moraceae } & Ficus religiosa & Seed & Feb, Mar, Apr & Feb, Mar, Apr \\
\hline & Ficus spp. 1 & Seed & Feb, Mar, Apr & Feb, Mar, Apr \\
\hline & Ficus spp. 2 (Bolus) & Seed & Jan & Jan, Apr \\
\hline & Morus nigra & Seed & Apr & Apr \\
\hline Myrtaceae & Psidium guajava & Seed & Jan, Feb & Jan, Feb \\
\hline Pteridophytes c.f. & & Flower & & Feb \\
\hline Tiliaceae & & Flower & & Feb \\
\hline
\end{tabular}

Myrtaceae, Moraceae, Malvaceae, Anarcadiaceae, Proteaceae, Pinaceae, and Rhamnaceae constitutes bat's diet in Kathmandu Valley. Seeds of Ficus religiosa, Morus nigra, $P$. gujava and other Ficus sps. were recorded in the P. giganteus diet. Ficus religiosa constitutes a higher portion at Kesharmahal and Morus nigra at Sallaghari. Similarly, pollens of Brassicaceae prevailed at both sites. Psidium guajava, Eucalyptus camaldulensis and Pinus roxburghii also occurred in high numbers (Fig. 2 and Fig. 3). The family Myrtaceae, Moraceae and Malvaceae have a higher frequency of occurrence in the bat's diet in both sites, Kesharmahal and Sallaghari. This study is similar to the preference of other fruit-eating bats in Tanzania, Australia, Pakistan and Myanmar (Entwistle \& Corp, 1997; Mahmood-Ul-Hassan et al., 2010; Parry-Jones \& Augee, 2001; Sharma, 2016; Win \& Mya, 2015). 




Figure 2 Frequency of occurrence of food items in P. giganteus diet in Kesharmahal

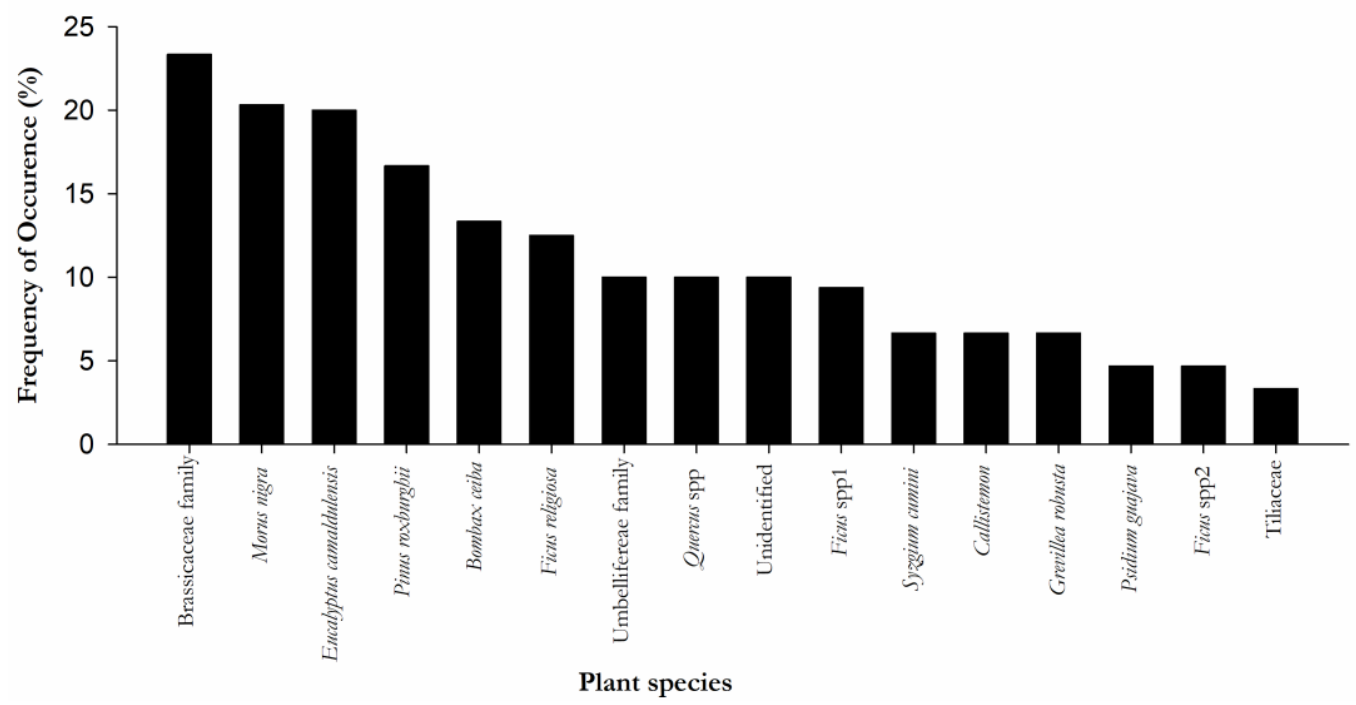

Figure 3 Frequency of occurrence of food items in $P$. gigantens diet in Sallaghari

The presence of seeds in droppings is comparatively lower than pollen. Seeds presence is lower in January and February and increases in March and April. According to Parry-Jones and Augee (2001), the occurrence of fruit in the droppings is lowest in winter and highest in spring and summer since fructification plants are majorly taken in spring and summer.

Pteropus giganteus is a canopy feeder. It feeds mostly fruits and flowers of trees rather than herbs. Brassicaceae and Chenopodiaceae occupy the key portion of the diet of $P$. giganteus, which are mainly herbs. It could be the reason due to the less availability of other food resources in the winter cold. Due to the shortage of food, the feeding behavior could have been changed from canopy feeder to ground feeder or might have feed taller spp. of these families.
Likewise, the reason could be that bat may want to gain many benefits (energy) by foraging in nearby locations using lower energy so that it can maximize fitness. Optimal Foraging Theory (OFT) best fits this strategy of a bat.

Since diet composition of $P$. giganteus is responsible for many factors. The ability to travel a longer distance and the availability of food nearby the roosting site (Parry-Jones \& Augee, 2001). The presence of Bombax ceiba in the P. giganteus diet evidence that it can travel a longer distance in search of food. Therefore, it is hard to predict the diet composition by the information of nearly by plant diversity.

Cutting of trees and branches for road expansion, construction of new buildings, electrocution, pollution and 
decreasing fruit farms have threatened the existence of flying foxes (Manandhar et al., 2017; Thapa, 2010). Degradation of habitat for both roosting sites and foraging is decreasing within Kathmandu Valley. Therefore, food source and habitat is shrinking for the survival of flying fox. Moreover, pollution, especially air pollution, might have adverse impacts on them, requiring further study. Bats are ecologically important species for pest control, dispersal of seed and pollination but people in Kathmandu Valley used to kill bats with medicine myths (Tuladhar-Douglas, 2008), religious superstition and protect their fruit from consumption (Jha, 2014). Therefore, planting fruit trees with diversity to nearby forests of the valley, conserving roosting sites, and other necessary actions should be taken to conserve bats in Kathmandu Valley.

\section{Conclusion}

Pteropus giganteus in Kathmandu Valley depends on plant families Myrtaceae (P. guajava, Callistemon spp., Syzygium cumini and E. camaldulensis), Brassicaceae, Malvaceae (Bombax ceiba) and Moraceae (Ficus spp., and Morus nigra) as a major food source from January to April. Pteropus giganteus in Kesharmahal is found to depend on 17 different plant families as a food source. Likewise, in Sallaghari, it depends on ten different plant families as a food source. Plant Families Apiaceae, Pinaceae (P. roxburghii), Fagaceae (Quercus $s p p$.), Proteaceae (Grevillea robusta) and Anacardiaceae (Mangifera indica) also contributes an important portion to the Pteropus diet. Seeds presence was noticed lower than that of flowers in the study periods in both study areas. The primary food item which forms the Pteropus diet is food items present nearby the roosting site. The presence of plants like Bombax ceiba proves that it can travel a longer distance searching for food. It can be said P. giganteus mostly depend on foods present nearby the roost and also flies far away in search of food, which is comparatively minimal. The presence of many pollens and seeds of different plant species confirms that $P$. giganteus plays a role in pollination and seed dispersion, including native and those of commercial fruit and crops. The findings suggest a need to preserve the habitat to provide for the long-term health and viability of $P$. giganteus.

\section{Acknowledgement}

The authors are thankful to Dr. Khum Narayan Poudel and Dr. Suresh Ghimire for their precious encouragement, guidance, and support and the Central Department of Environmental Science for providing facilities and financial support to conduct the research.

\section{References}

Acharya, P.R., Adhikari, H., Dahal, S., Thapa, A., \& Thapa, S. (2012). Bats of Nepal, A field guide. In Small Mammals Conservation and Research Foundation.

Aziz, S.A., Clemments, G.R., Peng, L.Y., Campos-Arceiz, A., McConkey, K.R., Forget, P., \& Gan, H.M. (2017). Elucidating the diet of the island flying fox (Pteropus bypomelanus) in Peninsular Malaysia through Illumina Next-Generation Sequencing. PeerJ, 5, e3176.

Aziz, S.A., Olival, K.J., Bumrungsri, S., Richards, G.C., \& Racey, P.A. (2016). The conflict between pteropodid bats and fruit growers: species, legislation and mitigation Bats in the Anthropocene: Conservation of bats in a changing world (pp. 377-426): Springer.

Entwistle, A., \& Corp, N. (1997). The diet of Pteropus voeltrkowi, an endangered fruit bat endemic to Pemba Island, Tanzania. African Journal of Ecology, 35(4), 351-360.

Fujita, M.S., \& Tuttle, M.D. (1991). Flying foxes (Chiroptera: Pteropodidae): threatened animals of key ecological and economic importance. Conservation Biology, 5(4), 455-463.

Ishtiaque, A., Shrestha, M., \& Chhetri, N. (2017). Rapid urban growth in the Kathmandu valley, Nepal: Monitoring land use land cover dynamics of a Himalayan city with Landsat imageries. Environments, 4(4), 72.

Jha, S.K. (2014). Findings on the Chiropteran status in Rautahat and Sarlahi, Nepal. The Initiation, 5, 50-54.

Jnawali, S.R., Baral H.S., Lee, S., Acharya, K.P., Upadhyay, G., Pandey, M., \& Amin, R. (2011). The Status of Nepal Mammals: The National Red List Series, Department of National Parks and Wildlife Conservation Kathmandu, Nepal.

Kasso, M., \& Balakrishnan, M. (2013). Ecological and economic importance of Bats (Order Chiroptera). ISRN Biodiversity, 2013, 1-9.

Koju, N., \& Chalise, M. (2010). Diurnal observation of population and general behavior of flying fox (Pteropus giganteus) in Sallaghari, Bhaktapur, Nepal. Journal of Natural History Museum, 25, 256-265.

Lewis, R.E., \& Harrison, D.L. (1962). Notes on bats from the Republic of Lebanon. Paper presented at the Proceedings of the Zoological Society of London.

Mahmood-Ul-Hassan, M., Gulraiz, T.L., Rana, S.A., \& Javid, A. (2010). The diet of Indian flying-foxes (Pteropus giganteus) in urban habitats of Pakistan. Acta Chiropterologica, 12(2), 341-347.

Manandhar, S., Thapa, S., Shrestha, T.K., Jyakhwo, R., Wright, W., \& Aryal, A. (2017). Population status and diurnal behaviour of the Indian flying fox Pteropus giganteus (Brünnich, 1782) in Kathmandu valley, Nepal. Proceedings of the Zoological Society. doi: 10.1007/s12595017-0219-x.

Mickleburgh, S.P., Hutson, A.M., \& Racey, P.A. (1992). Old World fruit bats. An action plan for their conservation. Gland, Switzerland: IUCN.

Muscarella, R., \& Fleming, T.H. (2007). The role of frugivorous bats in tropical forest succession. Biological Reviews, 82(4), 573-590.

Parry-Jones, K.A., \& Augee, M. (2001). Factors affecting the occupation of a colony site in Sydney, New South Wales by the Grey-headed Flying-fox Pteropus poliocephalus (Pteropodidae). Austral Ecology, 26(1), 47-55.

Sharma, B. ( 2016). Diet analysis of Indian flying fox (Pteropus giganteus Brunn. Pteropodidae) in sub-tropical mid-bills of Nepal (Unpublished thesis), Institute of Forestry, Pokhara, Nepal.

Srinivasulu, C., Racey, P.A., \& Mistry, S. (2010). A key to the bats (Mammalia: Chiroptera) of South Asia. Journal of Threatened Taxa, 2(7), 1001-1076.

Stier, S.C., \& Mildenstein, T.L. (2005). Dietary habits of the world's largest bats: the Philippine flying foxes, Acerodon 
jubatus and Pteropus vampyrus lanensis. Journal of Mammalogy, 86(4), 719-728.

Thapa, S. (2010). An updated checklist of valid bat species of Nepal. Small Mammal Mail-Bi-Annual Newsletter of CCINSA \& RISCINSA, 2(1), 16-17.

Thapa, S. (2014). A checklist of mammals of Nepal. Journal of Threatened Taxa, 6(8), 6061-6072.

Tuladhar-Douglas, W. (2008). The use of bats as medicine among the Newars. Journal of Ethnobiology, 28(1), 69-91.
Voigt, C., Kelm, D., Bradley, B., \& Ortmann, S. (2009). Dietary analysis of plant-visiting bats. In Kunz, T.H., \& Parsons, S. (Eds.), Ecological and Behavioral Methods for the Study of Bats. The Johns Hopkins University Press, Baltimore, pp. 593-609.

Win, S.S., \& Mya, K.M. (2015). The diet of the Indian flying fox Pteropus giganteus (Brünnich. 1782) (Chiroptera: Pteropodidae) in Myanmar- conflicts with local people? Journal of Threatened Taxa, 7(9), 7568-7572. 